\title{
La coda lunga degli eventi
}

\author{
Stefania DEMETZ*
}

\section{Gli eventi sono una bolla}

"Il mondo interno ai Giochi Olimpici assomiglia sempre più a una navicella spaziale, che in una lunga manovra di atterraggio, si adagia in un pianeta straniero, vi si ferma per un mese e poi riparte".

Scriveva così, a conclusione dei Giochi Olimpici Invernali di Sochi, il giornalista Christof Siemens sul settimanale tedesco Die Zeit. (Siemens, 2014)

I grandi eventi, ci dice questo articolo, sembrano essere divenuti qualcosa di sempre più estraneo alla realtà che li ospita. Standard internazionali per i servizi ai diversi "clienti" (atleti, stampa, televisioni, pubblico), "look" omologato nelle scenografie e nei linguaggi e sponsor globali di multinazionali che tendono a rendere uguali tra loro le competizioni, gli stadi, gli scenari a prescindere da dove abbiano luogo. La presenza del timbro locale si riduce spesso a un folclore incastrato dentro messaggi promozionali. Quando poi però la navicella lascia il terreno, il paesaggio cambia. Gli edifici vengono spogliati, le piazze tornano vuote, i souvenir finiscono nei retrobottega dei negozi.

È questo il momento della verità. Il momento in cui si comprende se quelle piazze, quelle strutture, la vita stessa all'interno di un territorio avranno un giovamento dal mega evento appena ospitato o se questo stesso territorio dovrà invece preoccuparsi di smaltire scorie imponenti.

Purtroppo i segni lasciati sono più spesso deludenti, piuttosto che gratificanti. Una volta spenti i riflettori, nessuno si occupa più di come riempire il vuoto e di come ridare un senso a luoghi che fino a poco prima hanno ospitato migliaia di persone, hanno accolto emozioni, hanno riempito giornali di storie, di immagini e di retorica. Spesso, gli stessi “concessionari” dell'evento se ne disinteressano.

Basta fare un tour virtuale in rete e trovare siti che raccontano cosa rimane. Le piscine di Atene 2004 sono arrugginite, (Smith, 2014) i trampolini di Torino 2006 sono decaduti (Lepore, 2014) e gli impianti costruiti, spesso con denaro pubblico, per Italia 90 sono in parte stati abbattuti (Reali, 2014).

\footnotetext{
* Direttrice generale delle gare di Coppa del Mondo di sci alpino in Val Gardena e-mail: stefi@ saslong.org
}

Sinergie, Italian Journal of Management

n. n. 95, Settembre-Dicembre 2014, pp. 185-194

ISSN 0393-5108 - DOI 10.7433/s95.2014.12 
Non deve pertanto meravigliare se a ogni occasione di un mega evento, si aprano polemiche che serbano una preoccupazione condivisa: Perché? Perché abbiamo ospitato questo circo temporaneo?

Il 2014 è un anno che non ci ha risparmiato da infinite e talvolta scomode polemiche: Sochi 2014 e Brasile 2014 hanno reso urgente più che mai una riflessione su eventi e sostenibilità, che oggi parrebbero due concetti incompatibili. Lo sono davvero?

Molti studi sembrano avvalorare questa tesi e ad esempio le posizioni severe di Cipra contro le Olimpiadi nelle Alpi sembrano non lasciare dubbi (Grass, 2014). E nemmeno i cahiers delle buone intenzioni, che sono richiesti, per esempio, dal Comitato Olimpico Internazionale, e in genere si chiamano "Piano di sostenibilità", sembrano bastare.

Forse allora si dovrebbe partire da un'altra prospettiva. Prima ancora di chiedersi perché organizzare un evento, ci si dovrebbe chiedere cosa sia un evento. Dovremmo cioè cercare, oltre l'apparenza, di identificarne le caratteristiche - si potrebbe dire - filosofiche, prima ancora che economiche. Dentro il contenitore evento c'è una potenza immensa, ma ci sono troppe potenziali fragilità. Solo dopo aver preso confidenza con le implicazioni possibili, si può passare alla fase successiva e chiedersi se si vuole organizzare un evento, che tipo di evento e soprattutto perché e con quale scopo.

\section{Caratteristiche dell'evento}

Partiamo dall'immagine esterna. Un evento è un raduno in uno spazio definito e in un periodo limitato che raccoglie migliaia e migliaia di persone. E festa e colori. È sorpresa e momenti di vita. È emozioni, passioni, fame, sete, freddo, caldo. È un ballare e cantare e un trepidare ed è farlo tutti insieme, tutti dentro una stessa ola che travolge. Un evento è una grande festa. È intrattenimento. E se poi sul palcoscenico ci va pure lo sport, diventa attesa, tensione, delusione, speranza.

È energia. E colori. E bandiere e sponsor, e marchi e pubblicità e televisione e giornalisti, e star, attori e pubblico. È una grande immensa cornice in cui gli spettatori oggi non sono più solo osservatori, ma sono attori a loro volta. E narratori a loro volta. Una grande carovana, un circo colorato, un sogno, un carnevale che passa e se ne va. Questo è un evento. Una grande esperienza collettiva.

La proliferazione di eventi parrebbe puntare soprattutto a questo aspetto: l'esperienza. Un concentrato di emozioni veicolate magistralmente. Gli organizzatori sempre piu divengono mediatori emozionali al servizio di un frammento di vita ${ }^{1}$. Ciò che conta è solo l'istante, quel momento. Il sociologo

1 Si veda a questo proposito "Consumare/investire il tempo libero" a cura di Massimo Lo Verde, in cui il tempo libero, il leisure è "guardato come uno dei processi attraverso cui vengono istituzionalizzati i luoghi e i tempi del contenimento delle emozioni” per cui un evento stesso diventa un'occasione di vita emozionale (Pepe, 2012). 
Michel Maffesoli scrive che le persone oggi "non sanno che farsene del fine da raggiungere, del progetto da realizzare: esse preferiscono «entrare nel» piacere di essere insieme, «entrare nell'» intensità del momento, «entrare nella» gioia di questo mondo com'è" (Maffesoli, 2004). Cioè ci dice che oggi le persone si radunano per un piacere assolutamente presente del godimento qui e ora, senza alcuna progettazione. Egli vede in questo una grande opportunità perché la voglia di condivisione è di per sé positiva. Se tuttavia questa opportunità è solo sfruttata per il momento, per il godimento o lo sfruttamento economico (o politico), allora questa società dell'ingresso può avere conseguenze devastanti e gli strascichi lasciati da tanti eventi sembrano dimostrarlo. Buone intenzioni iniziali, che poi il vortice della spettacolarità del momento fa sfumare.

Un evento allora non può spiegarsi solo in funzione di questo suo istante.

Se proviamo allora a guardarlo da una prospettiva diversa, non cioè dall'interno della cornice, ma da un ipotetico vista point alternativo, che ce ne mostri il paesaggio complessivo dentro un suo fluire temporale, troveremmo ben altre caratteristiche: quelle che troppo spesso dimentichiamo. È più facile lasciarsi incantare dalla punta di un iceberg colorato, piuttosto che guardare sotto, e cercare le fondamenta di questo iceberg.

Se infatti è vero che l'evento contiene in sé il senso dell'improvviso, della subitaneità, dell'istante, esso, tuttavia, è anche un far accadere, cioè non un istante ma un'integrazione di istanti legati in un processo. Scrive il filosofo Paul Ricoeur: «L'evento è ciò che fa sì che la successione di trasformazioni renda altra la situazione terminale rispetto a quella iniziale». È questa forse la considerazione chiave: agli estremi di un evento vi sono un prima e un dopo diversi tra loro. «Qualcosa che accade - dice Ricoeur - è qualcosa che passa e ciò che passa non si lascia pensare se non in rapporto a ciò che permane: la sostanza» (Ricoeur, 1990). Ciò significa che un evento non è solo l'istante, l'evento in sé, ma è anche la sua sostanza, ciò che rimane.

L'evento è l'intangibile che passa, ma al suo passaggio questo intangibile lascia delle impronte. Lo stadio che viene costruito non è l'evento. La medaglia d'oro non è l'evento. «Il corpo è ciò che esiste, mentre l'evento è il fare» ci dice Gilles Deleuze (Agostini, 2003). La medaglia è il corpo che rimane e l'evento è l'incorporeo che la realizza. Ed è la traccia di un accadimento cui si è dato un senso (la vittoria) dentro l'evento. E allo stadio che rimane che senso si vuole dare?

Ecco allora che pensare l'evento nel suo senso dinamico, e dunque come una tappa dentro una trasformazione, impone fin dalle prime riflessioni, fin dal quell'istante in cui nasce l'idea, un approccio complessivo più aperto e più consapevole.

\section{Sostenibilità dinamica}

Entra in gioco a questo punto il termine sostenibilità. Per capire, tuttavia, come legare un evento a un progetto di sostenibilità può essere utile un piccolo viaggio 
etimologico. Dal latino sus tenere, il senso originario da cui deriva "sostenibilità" rimanda a un "tenere sopra", da cui mantenere, conservare, nutrire. Ecco qui un primo scoglio. Se ci lasciassimo guidare, infatti, da sovrapposizioni semantiche, potremmo chiederci come sia possibile sos-tenere (cioè conservare) l'evento, cioè qualcosa che per sua natura è dinamico ed effimero. L'espressione legacy forse può offrire qualche supporto aggiuntivo. Il termine inglese significa in modo più chiaro eredità e in estensione "qualcosa lasciato da un predecessore". La parola si sviluppa dal latino legatus ovvero persona delegata, ambasciatore. Dunque la legacy di un evento può essere intesa come un'eredità lasciata a una comunità e a un territorio. È forse più simile all'equivalente tedesco Nachahaltigkeit (che similmente a sostenibilità significa conservare, dove però il "nach" indica un conservare dopo). Eppure anche in questi casi si perde il senso dinamico. Un evento passa, finisce, si conclude e allora cosa dobbiamo conservarne dopo? Fredmund Malik in un testo sulle parole pericolose del management invita a considerare una parola ben diversa: viability, vale a dire la vitalità.

Scrive Malik: "Viability è la capacità di salvaguardare una data funzionalità per un tempo illimitato. Per fare un esempio: se una certa risorsa è esaurita, un sistema deve essere in grado di spostarsi su un'altra risorsa. Deve prepararsi e tararsi per tempo, in modo da poter compiere il passaggio ( ...). È la capacità di evolvere". $\left(\right.$ Malik, 2004) ${ }^{2}$

La pianificazione di un evento deve quindi tenere conto di quale passaggio dovrà obbligatoriamente compiere. L'evento è per sua natura effimero. L'esaurimento delle risorse non è dunque una possibilità, ma un destino congenito e non posticipabile. Nel momento in cui scende il sipario o si spegne la fiamma olimpica inizia una nuova fase, come se si esaurisse la risorsa "evento" nel senso di raduno spazio-tempo, ma non la sostanza che questa risorsa ha prodotto.

Stadi, tribune centri stampa, trampolini, computer, carta, bicchieri, pentole, tavoli: che ne facciamo? E delle persone, del capitale umano impiegato durante la manifestazione ce ne preoccupiamo o lasciamo che ognuno vada per la propria strada $?^{3}$

\section{Perché organizziamo eventi}

A questo punto la domanda iniziale, una volta compreso che un evento è in ogni caso solo un passaggio e in ogni caso dovrà smaltire materiale, risorse, spazzatura, deve essere semplice ma diretta: Perché? E dovrebbe essere un perché che esprima sia il suo senso causale, sia quello finale.

2 La traduzione dal tedesco è propria.

Un esempio interessante di gestione dei collaboratori di un evento formati per la manifestazione e accompagnati poi in percorso successivo di inserimento del mondo del lavoro in ambito prevalentemente turistico è offerto dal festival della fotografia Rencontres di Arles (http://www.rencontres-arles.com) 
Ogni organizzatore dovrebbe partire da alcuni perché. E dovrebbe farlo tenendo bene in mente il concetto di viabilty, una linea guida preziosa per consentire a spazi e risorse, che hanno esaurito un loro ciclo dentro l'evento, una nuova vita dentro un processo di riciclo e di trasformazione. La responsabilità di un organizzatore sta tutta qui: essere certo che l'evento funzioni e che dopo vi si sia un riciclaggio funzionale efficace e ben pianificato. È interessante notare che in Germania, sebbene la parola Event, sia sempre più diffusa, il suo equivalente Veranstaltung resiste all'anglicismo. Veranstaltung, a differenza di manifestazione, sottende un senso che nella nostra "manifestazione" manca: la responsabilità. Anstalten significa organizzare e il suffisso Ver implica responsabilità. Dunque: Veranstaltung è organizzare in modo responsabile.

Un’organizzazione di eventi, dalla più semplice alla più complessa deve partire da qui: responsabilità per ciò che mette in scena e per ciò che dovrà vivere dopo.

Avviene ovviamente un passaggio di consegne: dall'organizzatore alla comunità ospitante. L'organizzatore, però, non finisce mai il suo ciclo di vita nel momento in cui cala il sipario. Il dopo, infatti, comprende una prima fase, quella propria dell'organizzatore, che dovrà chiudere senza danni la sua attività ${ }^{4}$. La seconda fase è quella della comunità, che dovrà saper sfruttare l'eredità ottenuta.

Un frequente malinteso, legato ai commenti e ai bilanci post evento è valutarne il successo sulla base quantitativa dell'evento in sé: quanti spettatori o quante televisioni o quanti palloncini si sono visti in cielo. Certamente uno stadio pieno significa che il marketing ha lavorato bene. Ma il vero successo di un evento lo si può misurare, nell'ottica di una lettura dinamica, solo dopo, solo quando la nuova vita del riciclo di risorse ha inizio. Non solo, il successo davvero lo si avrà solo quando lo scopo, vale a dire il motivo per cui si è deciso di ospitare un evento, sarà centrato. E allora è da questo inizio che si può partire.

Perché decidere di organizzare un evento?

Le motivazioni possono essere moltissime. In modo schematico si potrebbe dire che l'evento è uno strumento per intrattenere (e dunque fine a sé stesso), per crescere (far crescere un territorio, un'economia), per vendere (un prodotto, ma anche una destinazione turistica) e infine per educare, nel senso di formare, diffondere saperi o modelli etici.

Vediamoli nel dettaglio.

\subsection{Per divertire}

Il divertimento, si legge nei dizionari, solleva l'animo dalle fatiche quotidiane ed è svago e passatempo. È un atto volto a far star bene. Si può concordare che nella decisione di organizzare un evento non sia generalmente il divertimento la motivazione primaria. È tuttavia indubbio che lo spettatore decide di assistere a un

4 Il Comitato organizzatore dei Giochi Olimpici di Vancouver (2010) ha chiuso definitivamente la sua attività nel momento in cui ha raggiunto il pareggio di bilancio, senza lasciare strascichi (Chan, 2014). 
evento per divertirsi e svagarsi. Al suo volersi divertire si legano altre motivazioni, spesso in modo inconsapevole, come il manifestare la propria identità nella condivisione di una passione e nell'appartenenza a un gruppo (Di Betta, et al., 2012). L'entertainment, inoltre, è indiscutibilmente una delle variabili primarie nella valutazione del successo di un evento e come tale il divertimento oggi non è semplice effetto, ma diventa consapevole obiettivo. In sostanza il divertimento assume oggi un rilievo strategico, e pur non essendo in genere lo scopo principale, vi si deve legare in modo efficace. Il divertire però è evidentemente legato solo all'evento nella sua fase di esecuzione.

\subsection{Per crescere}

Crescere significa diventare maggiore, di quantità, di qualità, di durata, di intensità. Da un punto di vista educativo significa allevare. In realtà il termine "crescita" oggi per convenzione è quasi esclusivamente associato allo sviluppo economico e dunque assume connotazioni spesso negative, sinonimo di sfruttamento, danno ambientale, imbruttimento, precarietà. Eppure, citando Florence Nouville, autrice di un libretto appassionato sull'economia, crescita "è una bella parola, ed è anche un bel verbo: crescere e diventare più belli". (Nouville, 2010), Un esempio interessante di crescita intesa come miglioramento, soprattutto percettivo nei confronti di una nazione è stata la Coppa del Mondo di Calcio in Germania nel 2006. Se da un lato la capacità imprenditoriale, l'innovazione e le abilità tecniche e scientifiche di questa nazione sono sempre state riconosciute, da un punto di vista relazionale i tedeschi sono pregiudizialmente assimilati a una certa freddezza. Il motto scelto dagli organizzatori fu una risposta chiara a tale percezione: "The world as a guest with friends". La Germania voleva presentarsi al mondo come una nazione tollerante, cosmopolita, ricca di idee e amichevole. Nulla fu lasciato al caso. Vennero formati i dipendenti degli hotel (circa seimila persone), furono coinvolti i giovani in progetti di vario tipo che esaltassero questi valori, furono associate manifestazioni di beneficienza e di promozione dell'integrazione. Furono creati luoghi di incontro, in concerto con gli sponsor principali, per stimolare il pubblico a incontrarsi anche fuori dallo stadio. La Erlebniswelt, traducibile con "mondo di sensazioni" o di esperienze, di avventure ... (e che riporta all'importanza del divertimento evidenziato del paragrafo precedente) ne era il cuore fisico e concettuale. Il risultato è sancito dalle statistiche riportate dall'Anholt Nation Brands Index del 2006. La Germania migliorò la sua immagine scalando la vetta nei valori di tolleranza e affabilità (Maenning e Porsche, 2008). Non fu tutto merito del mega evento della FIFA, ma ciò non toglie che gli eventi, soprattutto quelli sportivi e forse soprattutto il calcio sono davvero strumenti unici per il loro effetto mediatico ed emotivo nella promozione di nazioni e territori. 


\subsection{Per vendere}

Nel marketing per la destinazione turistica l'evento diventa uno straordinario prodotto di promozione del territorio. Questo è oggi forse uno dei principali motivi che porta località turistiche a candidarsi a ospitare eventi, soprattutto sportivi. Va tenuto conto che ciò vale soprattutto per gli sport outdoor e soprattutto per quegli eventi che mettono in scena lo sport, prodotto primario della propria industria turistica. Lo sci e il ciclismo sembrano forse gli sport che meglio si adattano a tali strategie: la stessa pista da sci percorsa dai campioni, gli stessi passi scalati dai ciclisti diventano mete, talvolta addirittura mitiche, degli appassionati. Un evento praticato dentro uno stadio attira tifosi, non potenziali turisti o necessariamente praticanti di quel dato sport dentro quella struttura. Anche se sempre di più gli stadi, soprattutto all'estero, sono aperti a visite guidate e offrono servizi vari, dal museo della squadra allo shop, è superfluo dire che ovviamente qui non si tratta di promozione turistica. Per giocare con i paradossi: organizziamo le nostre vacanze sulle piste della Coppa del Mondo di sci, ma non di certo dentro gli stadi. È però vero che in concomitanza di una partita di calcio si può visitare anche la città che ospita quello stadio. Se però torniamo all'organizzatore, la società calcistica non organizza la partita per promuovere la propria città e gli effetti turistici sono solo collaterali all'evento in sé. Diverso, come si è visto, è il caso di un mega evento che coinvolga un'intera nazione, come la Coppa del Mondo di calcio in Germania nel 2006 di cui si è parlato nel paragrafo precedente.

Accade tuttavia che un evento sia talvolta messo in scena o che addirittura una disciplina sportiva sia "inventata" per promuovere un prodotto. L'esempio più eclatante oggi ce lo offre una bibita eccitante e dolciastra.

La RedBull ha superato ogni iniziale aspettativa in termini di crescita economica e lo ha fatto con un marketing aggressivo dentro lo sport. Legando il "carattere" dello sport alla propria bibita (dinamicità, sfida, divertimento) il fondatore ha deciso di lanciare il brand non semplicemente sponsorizzando in modo passivo eventi altrui, ma organizzando eventi propri come l'Airshow Flugtag ed il Red Bull Air Race o acquistando squadre (dalla scuderia di Formula uno al calcio). L'identificazione tra sport (spesso spericolato) e RedBull oggi è totalmente riuscita. Lo sport diventa in questo caso il medium principale di un'intera strategia aziendale.

\subsection{Per educare}

Ci sono eventi, sempre in ambito sportivo, che pongono l'educazione, la formazione, la diffusione di un'etica della condivisione e della compartecipazione in cima alla lista degli obiettivi. Esplicita certamente è questa intenzione nei tanti eventi "charity", che talvolta registrano un grande ritorno mediatico, si pensi ad esempio alla "Partita del Cuore" in Italia. È un dato di fatto, però, che nello sport rimane sempre più sbiadita questa intenzione. Sbiadita non significa esclusa, ma certamente nella scala delle priorità non è l'educazione a porsi in cima alla vetta $\mathrm{e}$ sovente essa una volta nominata si converte in uno straordinario strumento di ... 
marketing. I Giochi Olimpici di Londra del 2012 sono un buon esempio. Il motto "Inspire a Generation" mirava a portare i giovani a praticare più sport. I risultati sono ancora discordanti e probabilmente è presto per fare valutazioni solide (Gibson, 2013). Tuttavia, studi governativi mostrano segnali incoraggianti. (Mayor, 2013) Le Olimpiadi hanno portato nuovi impianti sportivi e un coinvolgimento emozionale sullo sport. Ma ciò non basta e solo una politica che sappia investire anche economicamente, in queste eredità potrà far si che lo scopo molto ambizioso venga raggiunto.

\section{Gestire con efficacia l'effimero}

Dentro questa ottica un evento è davvero solo un'occasione, uno strumento, un mezzo per raggiungere altro. Quale sia questo altro che si vuole raggiungere deve essere definito in modo chiaro fin dalle prime azioni. La navicella spaziale, di cui si è detto nell'incipit, non dovrebbe disorientare. La navicella, è vero, passa e se ne va, ma lascia una sostanza programmata che è compito poi degli eredi (amministrazioni pubbliche o società incaricate di gestire il lascito) saper far fruttare per il bene della collettività

Un evento va dunque amministrato anche nella sua fase di "coda lunga". Peter Drucker, il padre spirituale del management, cita una storia che può fungere da guida: "Uno scultore greco del 500 a.C. era stato incaricato di costruire una serie di statue da collocare in cima a un edificio. Lo scultore ci mise molto più del previsto perché voleva dare alla parte posteriore la stessa bellezza della parte anteriore. Le autorità, irritate per il ritardo, chiesero spiegazioni: «Perché ti occupi tanto della schiena, nessuno la vedrà!» e lui rispose: «Si, ma gli dei possono vederla»". (Ducker e Maciarello, 2013).

Il "fuggi-fuggi" del dopo evento è contrario a questa responsabilità. È un non occuparsi del retro delle statue. Management vuol dire in fondo definire obiettivi, organizzare, prendere decisioni e far crescere le persone (Malik, 2011) e dovrebbe valere anche a fine evento.

È una questione di prospettive. Un evento è, come si visto un insieme di un prima, un durante e un dopo, e il management deve agire responsabilmente in tutte queste tre fasi.

Fig. 1: Il management degli eventi

\begin{tabular}{|l|l|l|l|}
\hline \multirow{3}{*}{ Evento } & $\begin{array}{l}\text { vita prima } \\
\text { aspettativa }\end{array}$ & $\begin{array}{l}\text { vita durante } \\
\text { esperienza }\end{array}$ & $\begin{array}{l}\text { vita dopo } \\
\text { traccia }\end{array}$ \\
\hline \multirow{3}{*}{ Managament } & $\begin{array}{l}\text { Definire } \\
\text { obiettivi }\end{array}$ & $>$ \\
\cline { 2 - 4 } & Organizzare - Decidere - Controllare \\
\cline { 2 - 3 } & \multicolumn{2}{|l|}{ Formare collaboratori - risorse umane } \\
\hline
\end{tabular}

Fonte: elaborazioni proprie 
In fondo cambiano solo i pesi, i bilanciamenti. Il management del prima richiede ambizione per l'evento e lucidità strategica su due livelli: quello dell'esecuzione e quello dell'eredità.

Il management del durante va inteso prima di tutto come un fornitura di servizi (che siano intrattenimento, servizi stampa, e così via)

Il management del dopo impone un riciclaggio di funzione per mantenere viva l'eredità formulata come obiettivo nel perché iniziale.

\section{Bibliografia}

AGOSTINI F. (2003), Deleuze: evento e immanenza, Mimesis Transisti, Milano.

CHAN K. (2014), VANOC Breaks Even, to be dissolved 4 years later 2010 Winter Games $\begin{array}{lllllll}\text { VancityBuzz. } & - & 4 & \text { luglio } 2014 \quad-\quad 10 & \text { luglio } 2014\end{array}$ http://www.vancitybuzz.com/2014/07/vanoc-breaks-even-dissolved-4-years-2010olympic-winter-games/.

DI BETTA P., AMENTA C. (2012), "Lo spettatore della serie A di calcio: condivisione dell'esperienza allo stadio o isolamento davanti alla televisione?", in Lo Verde F.M., (a cura di) Consumare/Investire il tempo libero, Bruno Mondadori, Milano

DRUCKER P., MACIARELLO J., (2013), Un anno con Drucker, Etas, Milano.

GIBSON O. (2013), "London 2012 Olympic Games legacy questioned as young people shun sport", The Guardian - 12 dicembre 2012 - 8 luglio 2013 http://www.theguardian.com/sport/2013/dec/12/london-2012-olympic-legacy-youngpeople-shun-sport

GODIN S. (2009), Tribù, Sperling \& Kupfer, Milano.

GRASS S. (2014), "Dieci argomenti contro le Olimpiadi invernali sulle Alpi", Cipra - Vivere nelle Alpi - 10 aprile 2014 - 8 luglio 2014 - http://www.cipra.org/it/dossiers/giochiolimpici-invernali/dieci-argomenti-contro-le-olimpiadi-invernali-sulle-alpi

LEPORE F. (2014), “E Torino rottama gli impianti”, L'Espresso, 9 febbraio 2012 - 8 luglio 2014 - http://espresso.repubblica.it/attualita/cronaca/2012/02/09/news/e-torinorottama-gli-impianti-1.40231

MAENNIG W., PORSCHE M. (2008), "The feel-good effect at mega sports events. Reccomendations for public and private adminsitration informed by the experience of the FIFA World Cup 2006", Hamburg contemporary economic Discussion, n. 18.

MAFFESOLI M. (2004), "Il tempo delle tribù", Guerini e Associati, Milano.

MALIK F. (2004), Gefährliche Managementwörter, Frankfurter Allgemeine Buch, Frankfurt/Main.

MALIK F. (2011), Il management come professione, Sole24Ore, Milano.

MAYOR HM GOVERNEMNT (2013), The Inspired by 2012:The legacy from the London 2012 Olympic and Paralympic Games, Cabinet Office, London.

NOUVILLE F. (2010), Ho studiato economia e me ne pento, Bollati Boringhieri, Torino.

PEPE V. (2012), "Le emozioni del/nel leisure time. Una rassegna", in Lo Verde F.M., (a cura di) Consumare/Investire il tempo libero, Bruno Mondadori, Milano.

REALI G. (2014) Mondiali Italia '90. Sprechi, opere incompiute, mutui accesi al 2014 , Wikispesa - 2 giugno $2014 \quad-8$ luglio 2014 http://wikispesa.costodellostato.it/Mondiali_Italia_'90._Sprechi,_opere_incompiute, mutui_accesi_al_2014 
RICOEUR P. (1990) Evento e senso. Segno e evento nel pensiero contemporaneo, Edizioni Universitarie Jaca, Palermo.

SIEMENS C. (2014), Das Raumschiff Olympia fliegt weiter, Die Zeit - 23 febbraio 2014 - 8 luglio $2014 \quad$ - http://www.zeit.de/sport/2014-02/sotschi-bilanz-olympischewinterspiele

SMITH H. (2014), Athens 2004 Olympics: what happened after the athletes went home? The Guardian - 9 maggio $2012 \quad-8$ luglio 2014 http://www.theguardian.com/sport/2012/may/09/athens-2004-olympics-athleteshome. 